This is an electronic reprint of the original article. This reprint may differ from the original in pagination and typographic detail.

Author(s): Nuutinen, Juho

Title: $\quad$ The Besov capacity in metric spaces

Year: $\quad 2016$

Version:

Please cite the original version:

Nuutinen, J. (2016). The Besov capacity in metric spaces. Annales Polonici

Mathematici, 117(1), 59-78. https://doi.org/10.4064/ap3843-4-2016

All material supplied via JYX is protected by copyright and other intellectual property rights, and duplication or sale of all or part of any of the repository collections is not permitted, except that material may be duplicated by you for your research use or educational purposes in electronic or print form. You must obtain permission for any other use. Electronic or print copies may not be offered, whether for sale or otherwise to anyone who is not an authorised user. 


\title{
The Besov capacity in metric spaces
}

\author{
Juho NuUtinen (Jyväskylä)
}

\begin{abstract}
We study a capacity theory based on a definition of Hajłasz-Besov functions. We prove several properties of this capacity in the general setting of a metric space equipped with a doubling measure. The main results of the paper are lower bound and upper bound estimates for the capacity in terms of a modified Netrusov-Hausdorff content. Important tools are $\gamma$-medians, for which we also prove a new version of a Poincaré type inequality.
\end{abstract}

1. Introduction. In this paper, we study a metric version of the Besov capacity in a metric measure space $(X, d, \mu)$ with a doubling measure $\mu$. Different capacities in the metric setting have been studied previously, for example, in [BB], [B], GT], [HK], [KM], Leh] and [NS]. In the Euclidean setting, the Besov capacity has been studied, for example, in [A1], A2, $[\mathrm{AH}],[\mathrm{AHS}],[\mathrm{AX}],[\mathrm{D}],[\mathrm{HN}],[\mathrm{MX}],[\mathrm{N1}],[\mathrm{N2}],[\mathrm{N} 3]$ and [Sto]. Our definition of the Besov capacity is based on the pointwise definition of fractional $s$-gradients and the Hajłasz-Besov space $N_{p, q}^{s}(X), 0<s<\infty$ and $0<p, q \leq \infty$. This characterization for Besov spaces was first introduced in [KYZ and has recently been applied, for example, in [GKZ, [HIT, [HKT], [HT1] and [HT2]. The Hajłasz-Besov space $N_{p, q}^{s}(X)$ consists of $L^{p}-$ functions $u$ that have a fractional $s$-gradient with finite mixed $l^{q}\left(L^{p}(X)\right)$ norm. A sequence of nonnegative measurable functions $\left(g_{k}\right)_{k \in \mathbb{Z}}$ is a fractional s-gradient of $u$ if it satisfies the Hajłasz type pointwise inequality

$$
|u(x)-u(y)| \leq d(x, y)^{s}\left(g_{k}(x)+g_{k}(y)\right)
$$

for all $k \in \mathbb{Z}$ and almost all $x, y \in X$ with $2^{-k-1} \leq d(x, y)<2^{-k}$. We give the precise definitions and notation in Section 2, where we also prove two useful lemmas for the fractional $s$-gradients.

2010 Mathematics Subject Classification: Primary 31E05; Secondary 31B15.

Key words and phrases: Besov spaces, capacity, metric spaces.

Received 15 October 2015; revised 10 April 2016.

Published online *. 
In Section 3, we define the Besov capacity and prove its basic properties. These include monotonicity, a version of subadditivity and several convergence results. In particular, we apply the results to show that Hajłasz-Besov functions are quasicontinuous with respect to this capacity. Besov capacity has been studied previously in the metric setting in $[\mathrm{B}]$ and $[\mathrm{Co}$. However, in these papers only the case $p=q$ is considered in a less general metric space. Ahlfors $Q$-regularity, for example, is assumed in both papers. In a recent preprint [HKT], some of the results of our Section 3 are stated and a version of subadditivity is proved. We provide several new results and full proofs to the basic properties of the Besov capacity not proved in [HKT].

The $\gamma$-medians are extremely useful tools in the setting of Besov spaces, especially when $0<p \leq 1$ or $0<q \leq 1$. In our proofs, they take the place of integral averages. Medians behave similarly to the integral averages, but have the advantage that the function does not need to be locally integrable. In Section 4, we study some of the basic properties of the $\gamma$-medians that we later use in our proofs. One of the main results of this section is a new Sobolev-Poincaré type inequality for the $\gamma$-medians. For slightly different results, see [HKT] and [HT2]. Also, we recall the definition of discrete median convolutions and use them as tools to obtain Theorem 4.8, which says that for compact sets it is equivalent to consider only the locally Lipschitz admissible functions when calculating the capacity.

In Section 5, we study a modified version of the Netrusov-Hausdorff content. The Netrusov-Hausdorff content was introduced in $\mathbb{R}^{n}$ by Netrusov [N2], [N3]. It has also been studied, for example, in [A2] and [HN]. We modify the Euclidean definition to the metric setting, since in our case the dimension of the space $X$ need not be constant. Instead of summing over the powers of the radii $r_{j}$ of the balls in the covering, we sum over the measures of the balls in the covering divided by the values $\phi\left(r_{j}\right)$ of an increasing function $\phi$. Our main results are lower and upper bounds for the capacity in terms of the modified Netrusov-Hausdorff content (see Theorems 5.4 and 5.5).

\section{Notation and preliminaries}

2.1. Basic assumptions and notation. We assume that the triple $(X, d, \mu)$, denoted simply by $X$, is a metric measure space equipped with a metric $d$ and a Borel regular, doubling outer measure $\mu$, for which the measure of every ball is positive and finite. The doubling property means that there is a fixed constant $c_{d}>0$, called the doubling constant, such that

$$
\mu(B(x, 2 r)) \leq c_{d} \mu(B(x, r))
$$

for every ball $B(x, r)=\{y \in X: d(y, x)<r\}$, where $x \in X$ and $r>0$. 
We define the integral average of a locally integrable function $u$ over a set $A$ of positive and finite measure by

$$
u_{A}=f_{A} u d \mu=\frac{1}{\mu(A)} \int_{A} u d \mu .
$$

We denote by $\chi_{E}$ denote the characteristic function of a set $E \subset X$ and by $\overline{\mathbb{R}}$ the extended real numbers $[-\infty, \infty]$. We write $L^{0}(X)$ for the set of all measurable, almost everywhere finite functions $u: X \rightarrow \overline{\mathbb{R}}$. In general, $C$ is a positive constant whose value is not necessarily the same at each occurrence.

2.2. Fractional $s$-gradients and Hajłasz-Besov spaces. We define the Hajłasz-Besov space in terms of pointwise inequalities, as in [KYZ]. This characterization is motivated by the definition of a generalized gradient and of the Hajłasz-Sobolev space $M^{s, p}(X)$, given for $s=1, p \geq 1$ in [H] and for fractional scales in [Y]. There are also other definitions of Besov spaces in the metric setting. They have been studied, for example, in GKS, GKZ, [HMY], [KYZ, [MY], [SYY], [YZ].

Definition 2.1. Let $0<s<\infty$. A sequence $\left(g_{k}\right)_{k \in \mathbb{Z}}$ of nonnegative measurable functions is a fractional s-gradient of a function $u \in L^{0}(X)$ if there exists a set $E$ with $\mu(E)=0$ such that

$$
|u(x)-u(y)| \leq d(x, y)^{s}\left(g_{k}(x)+g_{k}(y)\right)
$$

for all $k \in \mathbb{Z}$ and all $x, y \in X \backslash E$ satisfying $2^{-k-1} \leq d(x, y)<2^{-k}$. The collection of all fractional $s$-gradients of $u$ is denoted by $\mathbb{D}^{s}(u)$.

We prove two lemmas that we use later. The above definition implies the following lattice property for fractional $s$-gradients.

Lemma 2.2. Let $0<s<\infty, u, v \in L^{0}(X),\left(g_{k}\right)_{k \in \mathbb{Z}} \in \mathbb{D}^{s}(u)$ and $\left(h_{k}\right)_{k \in \mathbb{Z}} \in \mathbb{D}^{s}(v)$. Then the sequence $\left(\max \left\{g_{k}, h_{k}\right\}\right)_{k \in \mathbb{Z}}$ is a fractional $s$ gradient of $\max \{u, v\}$ and $\min \{u, v\}$.

Proof. We define $w=\max \{u, v\}$ and assume that $G$ and $H$ are the exeptional sets for $\left(g_{k}\right)_{k \in \mathbb{Z}}$ and $\left(h_{k}\right)_{k \in \mathbb{Z}}$ in Definition 2.1. Clearly, the function $w$ is measurable and $\left(\max \left\{g_{k}, h_{k}\right\}\right)_{k \in \mathbb{Z}}$ is a sequence of nonnegative measurable functions. We show that (2.1) holds outside the set $G \cup H$ of measure zero. Let

$$
\begin{aligned}
& F_{u}=\{x \in X \backslash(G \cup H): u(x) \geq v(x)\}, \\
& F_{v}=\{x \in X \backslash(G \cup H): u(x)<v(x)\} .
\end{aligned}
$$

If $x, y \in F_{u}$ then

$$
|w(x)-w(y)|=|u(x)-u(y)| \leq d(x, y)^{s}\left(g_{k}(x)+g_{k}(y)\right)
$$


for all $k \in \mathbb{Z}$ satisfying $2^{-k-1} \leq d(x, y)<2^{-k}$. Similarly, for $x, y \in F_{v}$ we get

$$
|w(x)-w(y)| \leq d(x, y)^{s}\left(h_{k}(x)+h_{k}(y)\right)
$$

for all $k \in \mathbb{Z}$ satisfying $2^{-k-1} \leq d(x, y)<2^{-k}$.

If $x \in F_{u}$ and $y \in F_{v}$, then we can look at the two cases $u(x) \geq v(y)$ and $u(x)<v(y)$ separately. In the first case

$$
\begin{aligned}
|w(x)-w(y)| & =|u(x)-v(y)|=u(x)-v(y) \\
& \leq u(x)-u(y) \leq d(x, y)^{s}\left(g_{k}(x)+g_{k}(y)\right)
\end{aligned}
$$

for all $k \in \mathbb{Z}$ satisfying $2^{-k-1} \leq d(x, y)<2^{-k}$. In the second case

$$
|w(x)-w(y)|=v(y)-u(x) \leq v(y)-v(x) \leq d(x, y)^{s}\left(h_{k}(x)+h_{k}(y)\right)
$$

for all $k \in \mathbb{Z}$ satisfying $2^{-k-1} \leq d(x, y)<2^{-k}$. The case $x \in F_{v}$ and $y \in F_{u}$ follows by symmetry, and hence

$$
|w(x)-w(y)| \leq d(x, y)^{s}\left(\max \left\{g_{k}, h_{k}\right\}(x)+\max \left\{g_{k}, h_{k}\right\}(y)\right)
$$

for all $k \in \mathbb{Z}$ and all $x, y \in X \backslash(G \cup H)$ such that $2^{-k-1} \leq d(x, y)<2^{-k}$. The proof for the function $\min \{u, v\}$ follows along the same lines.

The next lemma is useful when we want to show that the supremum of countably many Hajłasz-Besov functions belongs to the Hajłasz-Besov space $N_{p, q}^{s}(X)$ (see Definition 2.4p.

Lemma 2.3. Let $u_{i} \in L^{0}(X)$ and $\left(g_{i, k}\right)_{k \in \mathbb{Z}} \in \mathbb{D}^{s}\left(u_{i}\right), i \in \mathbb{N}$, and define $u=\sup _{i \in \mathbb{N}} u_{i}$ and $\left(g_{k}\right)_{k \in \mathbb{Z}}=\left(\sup _{i \in \mathbb{N}} g_{i, k}\right)_{k \in \mathbb{Z}}$. If $u \in L^{0}(X)$, then $\left(g_{k}\right)_{k \in \mathbb{Z}} \in \mathbb{D}^{s}(u)$.

Proof. Since $u \in L^{0}(X)$, it is finite almost everywhere. Let $x, y \in X \backslash E$, with $u(y) \leq u(x)<\infty$, where $E$ is the union of exceptional sets for the functions $u_{i}$ in Definition 2.1. Let $\epsilon>0$. There is $i=i_{x} \in \mathbb{N}$ such that $u(x)<u_{i}(x)+\epsilon$. Now, since $u(y) \geq u_{i}(y)$, we have

$$
\begin{aligned}
|u(x)-u(y)| & =u(x)-u(y) \leq u_{i}(x)+\epsilon-u_{i}(y) \\
& \leq d(x, y)^{s}\left(g_{i, k}(x)+g_{i, k}(y)\right)+\epsilon \leq d(x, y)^{s}\left(g_{k}(x)+g_{k}(y)\right)+\epsilon
\end{aligned}
$$

for all $k \in \mathbb{Z}$ satisfying $2^{-k-1} \leq d(x, y)<2^{-k}$. Letting $\epsilon \rightarrow 0$ proves the claim.

For $0<p, q \leq \infty$ and a sequence $\left(f_{k}\right)_{k \in \mathbb{Z}}$ of measurable functions, we define

$$
\left\|\left(f_{k}\right)_{k \in \mathbb{Z}}\right\|_{l^{q}\left(L^{p}(X)\right)}=\left\|\left(\left\|f_{k}\right\|_{L^{p}(X)}\right)_{k \in \mathbb{Z}}\right\|_{l^{q}},
$$

where

$$
\left\|\left(a_{k}\right)_{k \in \mathbb{Z}}\right\|_{l q}= \begin{cases}\left(\sum_{k \in \mathbb{Z}}\left|a_{k}\right|^{q}\right)^{1 / q} & \text { when } 0<q<\infty \\ \sup _{k \in \mathbb{Z}}\left|a_{k}\right| & \text { when } q=\infty .\end{cases}
$$


Definition 2.4. Let $0<s<\infty$ and $0<p, q \leq \infty$. The homogeneous Hajtasz-Besov space $\dot{N}_{p, q}^{s}(X)$ consists of all functions $u \in L^{0}(X)$ for which the (semi)norm

$$
\|u\|_{\dot{N}_{p, q}^{s}(X)}=\inf _{\left(g_{k}\right) \in \mathbb{D}^{s}(u)}\left\|\left(g_{k}\right)\right\|_{l^{q}\left(L^{p}(X)\right)}
$$

is finite. The Hajtasz-Besov space $N_{p, q}^{s}(X)$ is $\dot{N}_{p, q}^{s}(X) \cap L^{p}(X)$ equipped with the norm

$$
\|u\|_{N_{p, q}^{s}(X)}=\|u\|_{L^{p}(X)}+\|u\|_{\dot{N}_{p, q}^{s}(X)} .
$$

For $0<s<1$ and $0<p, q \leq \infty$, the space $N_{p, q}^{s}\left(\mathbb{R}^{n}\right)$ coincides with the classical Besov space defined via differences ( $L^{p}$-modulus of smoothness, see [GKZ]). When $0<p<1$ or $0<q<1$, the (semi)norms defined above are actually quasi(semi)norms, but for simplicity we call them just norms. Recall that a quasinorm is similar to a norm in that it satisfies the norm axioms, except that there is a constant $C>1$ on the right-hand side of the triangle inequality.

2.3. Inequalities. We will often use the elementary inequality

$$
\sum_{i \in \mathbb{Z}} a_{i} \leq\left(\sum_{i \in \mathbb{Z}} a_{i}^{\beta}\right)^{1 / \beta},
$$

which holds whenever $a_{i} \geq 0$ for all $i$ and $0<\beta \leq 1$. Hölder's inequality for sums (when $1<b<\infty$ ) and 2.2 imply the next lemma that we use later to estimate the norms of fractional gradients.

Lemma 2.5 ([[HT, Lemma 3.1]). Let $1<a<\infty, 0<b<\infty$ and $c_{k} \geq 0$, $k \in \mathbb{Z}$. There exists a constant $C=C(a, b)$ such that

$$
\sum_{k \in \mathbb{Z}}\left(\sum_{j \in \mathbb{Z}} a^{-|j-k|} c_{j}\right)^{b} \leq C \sum_{j \in \mathbb{Z}} c_{j}^{b} .
$$

3. Capacity. In this section, we study a metric version of the Besov capacity. We prove its basic properties, including several useful lemmas and convergence results. In particular, we show that Hajłasz-Besov functions $u \in N_{p, q}^{s}(X), 0<s<1$ and $0<p, q<\infty$, are quasicontinuous with respect to this capacity (see Theorem 3.10). Recently, some of the results of this section have been stated or proved in [HKT]. We give complete proofs to the results not proved there as well as to new ones.

Definition 3.1. Let $0<s<\infty$ and $0<p, q \leq \infty$. The Besov capacity of a set $E \subset X$ is

$$
C_{p, q}^{s}(E)=\inf \left\{\|u\|_{N_{p, q}^{s}(X)}^{p}: u \in \mathcal{A}(E)\right\}
$$


where

$$
\mathcal{A}(E)=\left\{u \in N_{p, q}^{s}(X): u \geq 1 \text { in a neighbourhood of } E\right\}
$$

is the set of admissible functions for the capacity. We say that a property holds $C_{p, q}^{s}$-quasieverywhere if it holds outside a set of $C_{p, q}^{s}$-capacity zero.

REMARK 3.2. Lemma 2.2 implies that

$$
C_{p, q}^{s}(E)=\inf \left\{\|u\|_{N_{p, q}^{s}(X)}^{p}: u \in \mathcal{A}^{\prime}(E)\right\}
$$

where $\mathcal{A}^{\prime}(E)=\{u \in \mathcal{A}(E): 0 \leq u \leq 1\}$. Since $\mathcal{A}^{\prime}(E) \subset \mathcal{A}(E)$, we see that $C_{p, q}^{s}(E) \leq \inf \left\{\|u\|_{N_{p, q}^{s}(X)}^{p}: u \in \mathcal{A}^{\prime}(E)\right\}$. To prove the reverse inequality, let $\epsilon>0$ and let $u \in \mathcal{A}(E)$ be such that

$$
\|u\|_{N_{p, q}^{s}(X)}^{p} \leq C_{p, q}^{s}(E)+\epsilon
$$

Then $v=\max \{0, \min \{u, 1\}\} \in \mathcal{A}^{\prime}(E)$ and by Lemma 2.2 we have $\mathbb{D}^{s}(u)$ $\subset \mathbb{D}^{s}(v)$. Now

$$
\inf \left\{\|w\|_{N_{p, q}^{s}(X)}^{p}: w \in \mathcal{A}^{\prime}(E)\right\} \leq\|v\|_{N_{p, q}^{s}(X)}^{p} \leq\|u\|_{N_{p, q}^{s}(X)}^{p} \leq C_{p, q}^{s}(E)+\epsilon
$$

and letting $\epsilon \rightarrow 0$ yields the desired inequality.

REMARK 3.3. It follows immediately that

$$
\mu(E) \leq C_{p, q}^{s}(E)
$$

for every $E \subset X$. Indeed, let $u \in \mathcal{A}(E)$. Then there is an open set $U \supset E$ such that $u \geq 1$ in $U$. Hence

$$
\mu(E) \leq \mu(U) \leq\|u\|_{L^{p}(X)}^{p} \leq\|u\|_{N_{p, q}^{s}(X)}^{p}
$$

and taking the infimum over all $u \in \mathcal{A}(E)$ proves the inequality.

The $C_{p, q}^{s}$-capacity is generally not an outer measure. The definition clearly implies monotonicity, but the capacity is not necessarily subadditive. However, for practical purposes it is enough that the capacity satisfies (3.1) below for some $r>0$. Even in the Euclidean setting, countable subadditivity for the Besov capacity is known only when $p \leq q$ (see [A1]).

Theorem 3.4 ([HKT, Lemma 6.4]). Let $0<s<\infty$ and $0<p, q \leq \infty$. Then there are constants $C \geq 1$ and $0<r \leq 1$ such that

$$
C_{p, q}^{s}\left(\bigcup_{i \in \mathbb{N}} E_{i}\right)^{r} \leq C \sum_{i \in \mathbb{N}} C_{p, q}^{s}\left(E_{i}\right)^{r}
$$

for all sets $E_{i} \subset X, i \in \mathbb{N}$. Actually, 3.1 holds with $r=\min \{1, q / p\}$.

The Besov capacity is an outer capacity. This means that the capacity of a set $E \subset X$ can be obtained by approximating $E$ with open sets from the outside. 
LEMMA 3.5. The $C_{p, q}^{s}$-capacity is an outer capacity, that is,

$$
C_{p, q}^{s}(E)=\inf \left\{C_{p, q}^{s}(U): U \supset E, U \text { open }\right\}
$$

Proof. By monotonicity, $C_{p, q}^{s}(E) \leq \inf \left\{C_{p, q}^{s}(U): U \supset E, U\right.$ open $\}$. To obtain the reverse inequality, let $\epsilon>0$ and let $u \in \mathcal{A}(E)$ be such that

$$
\|u\|_{N_{p, q}^{s}(X)}^{p} \leq C_{p, q}^{s}(E)+\epsilon .
$$

Now, since $u$ is an admissible function for the capacity, there is an open set $U$ containing $E$ such that $u \geq 1$ on $U$. Then

$$
C_{p, q}^{s}(U) \leq\|u\|_{N_{p, q}^{s}(X)}^{p} \leq C_{p, q}^{s}(E)+\epsilon .
$$

Letting $\epsilon \rightarrow 0$ proves the claim.

The following compatibility condition says that removing a set of measure zero does not change the capacity of an open set. In particular, this can be applied to prove a uniqueness for $C_{p, q}^{s}$-quasicontinuous representatives of a Hajłasz-Besov function (see Remark 3.11.

LEMmA 3.6. Let $0<s<\infty$ and $0<p, q \leq \infty$. If $U$ is an open set and $\mu(E)=0$, then

$$
C_{p, q}^{s}(U)=C_{p, q}^{s}(U \backslash E) .
$$

Proof. Clearly, by monotonicity, $C_{p, q}^{s}(U) \geq C_{p, q}^{s}(U \backslash E)$ so it remains to show the other inequality. Let $\epsilon>0$ and let $u \in \mathcal{A}^{\prime}(U \backslash E)$, with $\left(g_{k}\right)_{k \in \mathbb{Z}}$ $\in \mathbb{D}^{s}(u)$, be such that $\chi_{U \backslash E} \leq u \leq 1$ and

$$
\left(\|u\|_{L^{p}(X)}+\left\|\left(g_{k}\right)\right\|_{l^{q}\left(L^{p}(X)\right)}\right)^{p}<C_{p, q}^{s}(U \backslash E)+\varepsilon .
$$

Let $v$ be a function such that $v=u$ in $X \backslash U$ and $v=1$ in $U$. Then $v=u$ outside the set $U \cap E$, which has measure zero, and so $\|v\|_{L^{p}(X)}=$ $\|u\|_{L^{p}(X)}$. Also, $\left(g_{k}\right)_{k \in \mathbb{Z}} \in \mathbb{D}^{s}(v)$, since we can choose the exceptional set in Definition 2.1 to be the union of $U \cap E$ and the exceptional set related to $u$ and $\left(g_{k}\right)_{k \in \mathbb{Z}}$. Then $v \in \mathcal{A}^{\prime}(U)$ and

$$
C_{p, q}^{s}(U) \leq\|v\|_{N_{p, q}^{s}(X)}^{p} \leq\left(\|u\|_{L^{p}(X)}+\left\|\left(g_{k}\right)\right\|_{l^{q}\left(L^{p}(X)\right)}\right)^{p}<C_{p, q}^{s}(U \backslash E)+\epsilon
$$

and letting $\epsilon \rightarrow 0$ proves the claim.

The outer capacity property of the Besov capacity implies the next convergence result for compact sets.9+ then

THEOREM 3.7. If $X \supset K_{1} \supset K_{2} \supset \cdots$ are compact sets and $K=\bigcap_{i=1}^{\infty} K_{i}$,

$$
\lim _{i \rightarrow \infty} C_{p, q}^{s}\left(K_{i}\right)=C_{p, q}^{s}(K)
$$

Proof. Clearly, by monotonicity, $\lim _{i \rightarrow \infty} C_{p, q}^{s}\left(K_{i}\right) \geq C_{p, q}^{s}(K)$ and so it remains to show the other inequality. If $U$ is an open set containing $K$, then 
$U \cup \bigcup_{i=1}^{\infty}\left(X \backslash K_{i}\right)$ is an open cover of $K_{1}$ and, since $K_{1}$ is compact, there is a finite subcover, i.e. a positive integer $N$ such that

$$
K_{1} \subset U \cup \bigcup_{i=1}^{N}\left(X \backslash K_{i}\right)=U \cup\left(X \backslash K_{N}\right) .
$$

It follows that $K_{N} \subset U$, since $K_{N} \subset K_{1}$. Hence, $\lim _{i \rightarrow \infty} C_{p, q}^{s}\left(K_{i}\right) \leq C_{p, q}^{s}(U)$ and by Lemma 3.5 we obtain

$$
\lim _{i \rightarrow \infty} C_{p, q}^{s}\left(K_{i}\right) \leq \inf \left\{C_{p, q}^{s}(U): U \supset K, U \text { open }\right\}=C_{p, q}^{s}(K) .
$$

We apply the following theorem to show that Hajłasz-Besov functions are quasicontinuous with respect to the $C_{p, q}^{s}$-capacity (see Theorem 3.10).

Theorem 3.8. Let $0<s<\infty$ and $0<p, q \leq \infty$. If $\left(u_{i}\right)_{i \in \mathbb{N}}$ is a Cauchy sequence of continuous functions in $N_{p, q}^{s}(X)$, then there is a subsequence of $\left(u_{i}\right)_{i \in \mathbb{N}}$ which converges pointwise $C_{p, q}^{s}$-quasieverywhere in $X$. Moreover, the convergence is uniform outside a set of arbitrary small $C_{p, q^{-}}^{s}$ capacity.

Proof. Let $r=\min \{1, q / p\}$. There is a subsequence of $\left(u_{i}\right)_{i \in \mathbb{N}}$, which we still denote by $\left(u_{i}\right)_{i \in \mathbb{N}}$, such that

$$
\sum_{i=1}^{\infty} 2^{i p r}\left\|u_{i}-u_{i+1}\right\|_{N_{p, q}^{s}(X)}^{p r}<\infty
$$

For $i, j \in \mathbb{N}$, let

$$
A_{i}=\left\{x \in X:\left|u_{i}(x)-u_{i+1}(x)\right|>2^{-i}\right\} \quad \text { and } \quad B_{j}=\bigcup_{i=j}^{\infty} A_{i} .
$$

Since the functions $u_{i}$ are continuous, the sets $A_{i}$ and $B_{j}$ are open. It follows that the function $2^{i}\left|u_{i}-u_{i+1}\right|$ is admissible for the Besov capacity of $A_{i}$ and

$$
C_{p, q}^{s}\left(A_{i}\right) \leq 2^{i p}\left\|u_{i}-u_{i+1}\right\|_{N_{p, q}^{s}(X)}^{p} .
$$

Now, by Theorem 3.4 .

$$
C_{p, q}^{s}\left(B_{j}\right) \leq C\left(\sum_{i=j}^{\infty} C_{p, q}^{s}\left(A_{i}\right)^{r}\right)^{1 / r} \leq C\left(\sum_{i=j}^{\infty} 2^{i p r}\left\|u_{i}-u_{i+1}\right\|_{N_{p, q}^{s}(X)}^{p r}\right)^{1 / r} .
$$

Since $B_{1} \supset B_{2} \supset \cdots$ and the sum $(3.2)$ converges, we have

$$
C_{p, q}^{s}\left(\bigcap_{j=1}^{\infty} B_{j}\right) \leq \lim _{j \rightarrow \infty} C_{p, q}^{s}\left(B_{j}\right)=0
$$

and $\left(u_{i}\right)_{i \in \mathbb{N}}$ converges pointwise in $X \backslash \bigcap_{j=1}^{\infty} B_{j}$. Moreover,

$$
\left|u_{j}(x)-u_{k}(x)\right| \leq \sum_{i=j}^{k-1}\left|u_{i}(x)-u_{i+1}(x)\right| \leq \sum_{i=j}^{k-1} 2^{-i} \leq 2^{1-j}
$$


for all $x \in X \backslash B_{j}$ and every $k>j$. Hence, the convergence is uniform in $X \backslash B_{j}$ and the claim follows.

Definition 3.9. A function $u: X \rightarrow \overline{\mathbb{R}}$ is $C_{p, q}^{s}$-quasicontinuous if for every $\varepsilon>0$ there exists a set $U$ such that $C_{p, q}^{s}(U)<\varepsilon$ and the restriction of $u$ to $X \backslash U$ is continuous.

Note that, by Lemma 3.5, the set $U$ can be chosen to be open.

TheOREM 3.10. Let $0<s<1$ and $0<p, q<\infty$. Then, for every $u \in N_{p, q}^{s}(X)$, there exists a $C_{p, q}^{s}$-quasicontinuous function $v$ such that $u=v$ almost everywhere.

Proof. Since continuous functions are dense in $N_{p, q}^{s}(X)$ when $0<s<1$ and $0<p, q<\infty$ (see [HKT, Theorem 1.1]), and $N_{p, q}^{s}(X)$ is complete by [HT2, appendix], the claim follows from the previous theorem. Indeed, $u \in N_{p, q}^{s}(X)$ if and only if there is a sequence $\left(u_{i}\right)_{i \in \mathbb{N}}$ of continuous functions in $L^{p}(X)$ and $\left(g_{i, k}\right)_{k \in \mathbb{Z}} \in \mathbb{D}^{s}\left(u_{i}-u\right)$ such that $u_{i} \rightarrow u$ in $L^{p}(X)$ and $\left\|\left(g_{i, k}\right)_{k \in \mathbb{Z}}\right\|_{l^{q}\left(L^{p}(X)\right)} \rightarrow 0$. By the previous theorem, the limit function is $C_{p, q}^{s}$-quasicontinuous.

REMARK 3.11. The $C_{p, q}^{s}$-quasicontinuous representative is unique in the sense that if two $C_{p, q}^{s}$-quasicontinuous functions coincide almost everywhere, then they actually coincide outside a set of $C_{p, q}^{s}$-capacity zero. This follows from Lemmas 3.5 and 3.6 , and from a nice argument, in an abstract setting, in $[\mathrm{K}]$.

4. $\gamma$-median. In this section, we study $\gamma$-medians, which are important tools in our setting of Besov spaces. In our proofs, they take the place of integral averages and are extremely useful when $0<p \leq 1$ or $0<q \leq 1$. One of the main results is Theorem 4.5 which is a new Sobolev-Poincaré type inequality for the medians. Recently, slightly different results have been proved in [HKT] and [HT2], where an additional nonempty spheres property is assumed on the underlying space $X$. In the last part of this section, we define a discrete median convolution which we use to show that it is equivalent to consider only the locally Lipschitz admissible functions when calculating the capacity of a compact set (see Theorem 4.8). These results are useful in Section 5, where we study a modified Netrusov-Hausdorff content related to the capacity.

Now, we define the $\gamma$-median of a function $u \in L^{0}(X)$ over a set of finite measure. Previously, the $\gamma$-medians have been studied, for example, in [F], [GKZ], [JPW], [JT], [Ler], [LP], [PT], [Str], [Z], and recently in [HIT], [HKT] and [HT2]. 
Definition 4.1. Let $0<\gamma \leq 1 / 2$. The $\gamma$-median of a function $u \in L^{0}(X)$ over a set $A$ of finite measure is

$$
m_{u}^{\gamma}(A)=\inf \{a \in \mathbb{R}: \mu(\{x \in A: u(x)>a\})<\gamma \mu(A)\} .
$$

In the following lemma, we give some basic properties of the $\gamma$-median.

Lemma 4.2. Let $A \subset X$ be a set with $\mu(A)<\infty$. Let $u, v \in L^{0}(A)$ and let $0<\gamma \leq 1 / 2$. The $\gamma$-median has the following properties:

(a) If $\gamma \leq \gamma^{\prime}$, then $m_{u}^{\gamma}(A) \geq m_{u}^{\gamma^{\prime}}(A)$.

(b) If $u \leq v$ almost everywhere, then $m_{u}^{\gamma}(A) \leq m_{v}^{\gamma}(A)$.

(c) If $A \subset B$ and $\mu(B) \leq C \mu(A)$, then $m_{u}^{\gamma}(A) \leq m_{u}^{\gamma / C}(B)$.

(d) If $c \in \mathbb{R}$, then $m_{u}^{\gamma}(A)+c=m_{u+c}^{\gamma}(A)$.

(e) If $c \in \mathbb{R}$, then $m_{c u}^{\gamma}(A)=c m_{u}^{\gamma}(A)$.

(f) $\left|m_{u}^{\gamma}(A)\right| \leq m_{|u|}^{\gamma}(A)$.

(g) For every $p>0$ and $u \in L^{p}(A)$,

$$
m_{|u|}^{\gamma}(A) \leq\left(\gamma^{-1} f_{A}|u|^{p} d \mu\right)^{1 / p} .
$$

(h) If $u$ is continuous, then

$$
\lim _{r \rightarrow 0} m_{u}^{\gamma}(B(x, r))=u(x) \quad \text { for every } x \in X .
$$

Proof. We prove the property (g) below. The other, quite straightforward proofs are left for the reader, who can also look at $[\mathrm{PT}]$ where most of the properties are proved in the Euclidean space. The proofs in the metric setting follow essentially the same lines.

For (g), we may assume that $m_{|u|}^{\gamma}(A) \neq 0$, since otherwise the claim is obvious. Let $p>0$ and $u \in L^{p}(A)$. The definition of the $\gamma$-median clearly implies that

$$
\gamma \mu(A) \leq \mu\left(\left\{x \in A:|u(x)| \geq m_{|u|}^{\gamma}(A)\right\}\right)=\mu\left(\left\{x \in A:|u(x)|^{p} \geq m_{|u|}^{\gamma}(A)^{p}\right\}\right)
$$

and by Chebyshev's inequality

$$
\mu\left(\left\{x \in A:|u(x)|^{p} \geq m_{|u|}^{\gamma}(A)^{p}\right\}\right) \leq \frac{1}{m_{|u|}^{\gamma}(A)^{p}} \int_{A}|u|^{p} d \mu .
$$

The claim follows by combining the above two estimates.

We have the following definition, analogous to the definition of a Lebesgue point of a function, when taking the limit of medians.

Definition 4.3. Let $u \in L^{0}(A)$. A point $x$ is a generalized Lebesgue point of $u$ if

$$
\lim _{r \rightarrow 0} m_{u}^{\gamma}(B(x, r))=u(x) \quad \text { for all } 0<\gamma \leq 1 / 2 .
$$


REMARK 4.4. Recently, it was shown in [HKT, Theorem 1.2] that every point outside of a set of $C_{p, q}^{s}$-capacity zero of a Hajłasz-Besov function $u$ is a generalized Lebesgue point of $u$ and that the limit of medians gives a $C_{p, q}^{s}$-quasicontinuous representative of the function. This is proved in [HKT] by defining a median maximal function and using it as a tool. In particular, a capacitary weak type estimate for the median maximal function is used in the proof.

Definition 2.1 of fractional $s$-gradients implies various Sobolev-Poincaré type inequalities for medians. Slightly different results than the following can be found, for example, in [HKT] and [HT2]. We obtain the next theorem even without assuming a nonempty spheres property, unlike in [HKT] and [HT2].

Theorem 4.5. Let $0<\gamma \leq 1 / 2,0<s, p<\infty$ and $0<q \leq \infty$. Let $u \in N_{p, q}^{s}(X)$. Then there is a constant $C>0$ and a sequence $\left(g_{k}\right)_{k \in \mathbb{Z}} \in \mathbb{D}^{s}(u)$ such that

$$
\inf _{c \in \mathbb{R}} m_{|u-c|}^{\gamma}\left(B\left(x, 2^{-k}\right)\right) \leq C 2^{-k s}\left(f_{B\left(x, 2^{-k+1}\right)} g_{k}^{p} d \mu\right)^{1 / p}
$$

for every $x \in X$ and $k \in \mathbb{Z}$. In fact, given any $\left(h_{j}\right)_{j \in \mathbb{Z}} \in \mathbb{D}^{s}(u)$, we can choose

$$
g_{k}=\left(\sum_{j \geq k-2} 2^{(k-j) s^{\prime} \tilde{p}} h_{j}^{p}\right)^{1 / p},
$$

where $0<s^{\prime}<s$ and $\tilde{p}=\min \{1, p\}$. Moreover, there is a constant $c>0$ such that

$$
\left\|\left(g_{k}\right)\right\|_{l^{q}\left(L^{p}(X)\right)} \leq c\left\|\left(h_{j}\right)\right\|_{l^{q}\left(L^{p}(X)\right)} .
$$

Proof. Let $\left(h_{j}\right)_{j \in \mathbb{Z}} \in \mathbb{D}^{s}(u)$. By [GKZ, Lemma 2.1] and by Lemma 4.2 (g), there exist constants $C>0$ and $0<s^{\prime}<s$ such that

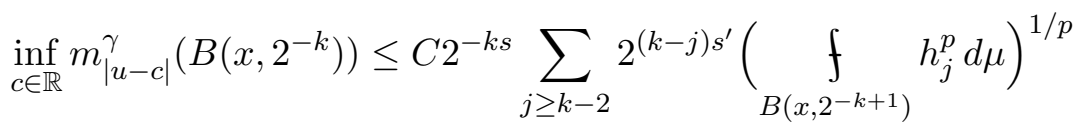

for every $x \in X$ and $k \in \mathbb{Z}$. We show that the right-hand side is bounded by $C 2^{-k s}\left(f_{B\left(x, 2^{-k+1}\right)} g_{k}^{p} d \mu\right)^{1 / p}$, where

$$
g_{k}=\left(\sum_{j \geq k-2} 2^{(k-j) s^{\prime} \tilde{p}} h_{j}^{p}\right)^{1 / p}
$$

and $\tilde{p}=\min \{1, p\}$. Notice that $\left(g_{k}\right)_{k \in \mathbb{Z}} \in \mathbb{D}^{s}(u)$, since

$$
|u(x)-u(y)| \leq d(x, y)^{s}\left(h_{k}(x)+h_{k}(y)\right) \leq d(x, y)^{s}\left(g_{k}(x)+g_{k}(y)\right)
$$

for all $k \in \mathbb{Z}$ and all $x, y \in X \backslash E$ satisfying $2^{-k-1} \leq d(x, y)<2^{-k}$. 
If $p>1$, we use Hölder's inequality for sums $\left(1 / p+1 / p^{\prime}=1\right)$ :

$$
\begin{aligned}
\sum_{j \geq k-2} 2^{(k-j) s^{\prime} / p^{\prime}} & 2^{(k-j) s^{\prime} / p}\left(f_{B\left(x, 2^{-k+1}\right)} h_{j}^{p} d \mu\right)^{1 / p} \\
& \leq\left(\sum_{j \geq k-2} 2^{(k-j) s^{\prime}}\right)^{1 / p^{\prime}}\left(\sum_{j \geq k-2} 2^{(k-j) s^{\prime}} f_{B\left(x, 2^{-k+1}\right)} h_{j}^{p} d \mu\right)^{1 / p} \\
& \leq C\left(f_{B\left(x, 2^{-k+1}\right)} \sum_{j \geq k-2} 2^{(k-j) s^{\prime}} h_{j}^{p} d \mu\right)^{1 / p},
\end{aligned}
$$

and if $0<p \leq 1$, by inequality 2.2 ,

$$
\begin{aligned}
\sum_{j \geq k-2} 2^{(k-j) s^{\prime}}\left(f_{B\left(x, 2^{-k+1}\right)} h_{j}^{p} d \mu\right)^{1 / p} & \leq\left(\sum_{j \geq k-2} 2^{(k-j) s^{\prime} p} f_{B\left(x, 2^{-k+1}\right)} h_{j}^{p} d \mu\right)^{1 / p} \\
& =\left(f_{B\left(x, 2^{-k+1}\right)} \sum_{j \geq k-2} 2^{(k-j) s^{\prime} p} h_{j}^{p} d \mu\right)^{1 / p} .
\end{aligned}
$$

Combining the two cases, we obtain 4.1.

To prove 4.3), we first see that

$$
\left\|g_{k}\right\|_{L^{p}(X)}^{p}=\int_{X} \sum_{j \geq k-2} 2^{(k-j) s^{\prime} \tilde{p}} h_{j}^{p} d \mu=\sum_{j \geq k-2} 2^{(k-j) s^{\prime} \tilde{p}}\left\|h_{j}\right\|_{L^{p}(X)}^{p} .
$$

Now, by Lemma 2.5, we get

$$
\sum_{k \in \mathbb{Z}}\left\|g_{k}\right\|_{L^{p}(X)}^{q} \leq \sum_{k \in \mathbb{Z}}\left(\sum_{j \geq k-2} 2^{(k-j) s^{\prime} \tilde{p}}\left\|h_{j}\right\|_{L^{p}(X)}^{p}\right)^{q / p} \leq C \sum_{j \in \mathbb{Z}}\left\|h_{j}\right\|_{L^{p}(X)}^{q},
$$

and consequently

$$
\left\|\left(g_{k}\right)\right\|_{l^{q}\left(L^{p}(X)\right)} \leq C\left\|\left(h_{j}\right)\right\|_{l^{q}\left(L^{p}(X)\right)} .
$$

Remark 4.6. Let $A \subset X$ be a set with $\mu(A)<\infty, u \in L^{0}(A)$ and $0<\gamma \leq 1 / 2$. Then

$$
m_{\left|u-m^{\gamma}(A)\right|}^{\gamma}(A) \leq 2 \inf _{c \in \mathbb{R}} m_{|u-c|}^{\gamma}(A),
$$

since for all $c \in \mathbb{R}$,

$$
\begin{aligned}
m_{\left|u-m^{\gamma}(A)\right|}^{\gamma}(A) & \leq m_{|u-c|+\left|c-m^{\gamma}(A)\right|}^{\gamma}(A)=m_{|u-c|}^{\gamma}(A)+\left|c-m_{u}^{\gamma}(A)\right| \\
& \leq m_{|u-c|}^{\gamma}(A)+m_{|u-c|}^{\gamma}(A),
\end{aligned}
$$

where we have used properties (b), (d) and (f) of $\gamma$-median from Lemma 4.2 .

Next, we define a discrete $\gamma$-median convolution that we use in the proof of Theorem 4.8. Discrete convolutions are standard tools in analysis on metric measure spaces (see, for example, $[\mathrm{CW}]$ and $[\mathrm{MS}]$ ) and they are used, for 
example, to define a discrete maximal function, introduced in [KL]. Analogously, a discrete $\gamma$-median maximal function can be defined by taking a supremum of the discrete $\gamma$-median convolutions (see, for example, [HKT]).

We fix a scale $r>0$ and cover the space $X$ with a countable family of balls $\left\{B_{i}\right\}=\left\{B\left(x_{i}, r\right)\right\}$, so that the enlarged balls are of bounded overlap. This means that there is a constant $C\left(c_{d}\right)>0$, depending only on the doubling constant, such that

$$
\sum_{i=1}^{\infty} \chi_{2 B_{i}}(x) \leq C\left(c_{d}\right)<\infty
$$

for all $x \in X$. Then a partition of unity related to the covering $\left\{B_{i}\right\}$ is constructed. There exist $C / r$-Lipschitz functions $\varphi_{i}, i=1,2, \ldots$, such that $0 \leq \varphi_{i} \leq 1, \varphi_{i}=0$ outside $2 B_{i}$ and $\varphi_{i} \geq C^{-1}$ on $B_{i}$ for all $i$ and $\sum_{i=1}^{\infty} \varphi_{i}=1$. Let $0<\gamma \leq 1 / 2$. A discrete $\gamma$-median convolution of a function $u \in L^{0}(X)$ at scale $r>0$ is

$$
u_{r}^{\gamma}(x)=\sum_{i=1}^{\infty} m_{u}^{\gamma}\left(B_{i}\right) \varphi_{i}(x)
$$

for all $x \in X$, where the balls $B_{i}$ and functions $\varphi_{i}$ are as above.

We apply the next theorem, by which locally Lipschitz functions are dense in $N_{p, q}^{s}(X)$, to show that for compact sets we can restrict the set of admissible functions in the definition of the $C_{p, q}^{s}$-capacity to locally Lipschitz functions when $0<s<1$ and $0<p, q<\infty$.

TheOREM 4.7 ([HKT, Theorem 1.1]). Let $0<\gamma \leq 1 / 2,0<s<1$, $0<p, q<\infty$ and $u \in \dot{N}_{p, q}^{s}(X)$. Then the discrete $\gamma$-median convolution approximations $u_{2^{-i}}^{\gamma}$ converge to $u$ in $N_{p, q}^{s}(X)$ as $i \rightarrow \infty$.

Theorem 4.8. Let $0<s<1,0<p, q<\infty$ and let $K \subset X$ be a compact set. Then

$$
C_{p, q}^{s}(K) \approx \inf \left\{\|u\|_{N_{p, q}^{s}(X)}^{p}: u \in \tilde{\mathcal{A}}(K)\right\},
$$

where $\tilde{\mathcal{A}}(K)=\{u \in \mathcal{A}(K): u$ is locally Lipschitz $\}$.

Proof. Since $\tilde{\mathcal{A}}(K) \subset \mathcal{A}(K)$, it suffices to prove the " $\geq$ " part. Let $u \in$ $\mathcal{A}(K)$. Then there is an open set $U \supset K$ such that $u \geq 1$ in $U$. Let $V=$ $\{x: d(x, K)<d(K, X \backslash U) / 2\}$. If $x \in V$ and $r<d(K, X \backslash U) / 8$, then $B(y, 2 r) \subset U$ whenever $x \in B(y, 2 r)$. It follows that $u_{r}^{\gamma} \geq 1$ in $V$ when $r<d(K, X \backslash U) / 8$. Thus, $u_{r}^{\gamma} \in \tilde{\mathcal{A}}(K)$ for small $r$, and so, by Theorem 4.7 ,

$$
\begin{aligned}
\inf \left\{\|v\|_{N_{p, q}^{s}(X)}^{p}: v \in \tilde{\mathcal{A}}(K)\right\} & \leq \liminf _{i \rightarrow \infty}\left\|u_{2^{-i}}^{\gamma}\right\|_{N_{p, q}^{s}(X)}^{p} \\
& \leq \liminf _{i \rightarrow \infty} C\left(\|u\|_{N_{p, q}^{s}(X)}^{p}+\left\|u_{2^{-i}}^{\gamma}-u\right\|_{N_{p, q}^{s}(X)}^{p}\right) \\
& \leq C\|u\|_{N_{p, q}^{s}(X)}^{p} .
\end{aligned}
$$

The claim follows by taking the infimum over $u \in \mathcal{A}(K)$. 
5. Netrusov-Hausdorff content. In this section, we define a modified version of the Netrusov-Hausdorff content and prove lower bounds and upper bounds for the Besov capacity in terms of the related NetrusovHausdorff cocontent. The Netrusov-Hausdorff content was first used by Netrusov [N2], [N3] when studying the relations between capacities and Hausdorff contents in $\mathbb{R}^{n}$. We modify this content by taking the sum over the measures of the balls in the covering divided by the values $\phi\left(r_{j}\right)$ of the radii, where $\phi$ is an increasing function. In the setting of a doubling metric measure space, this kind of modification, instead of summing the powers of the radii of the balls in the covering, is natural since the dimension of the space is usually not (even locally) constant.

Definition 5.1. Let $\phi:(0, \infty) \rightarrow(0, \infty)$ be an increasing function and let $0<\theta<\infty$ and $0<R<\infty$. The Netrusov-Hausdorff cocontent of a set $E \subset X$ is

$$
\mathcal{H}_{R}^{\phi, \theta}(E)=\inf \left[\sum_{i: 2^{-i}<R}\left(\sum_{j \in I_{i}} \frac{\mu\left(B\left(x_{j}, r_{j}\right)\right)}{\phi\left(r_{j}\right)}\right)^{\theta}\right]^{1 / \theta},
$$

where the infimum is taken over all coverings $\left\{B\left(x_{j}, r_{j}\right)\right\}$ of $E$ with $0<r_{j} \leq R$ and $I_{i}=\left\{j: 2^{-i} \leq r_{j}<2^{-i+1}\right\}$. When $R=\infty$, the infimum is taken over all coverings of $E$ and the first sum is over $i \in \mathbb{Z}$. When $\phi(t)=t^{d}$, we use the notation $\mathcal{H}_{R}^{d, \theta}:=\mathcal{H}_{R}^{\phi, \theta}$.

Notice that if the measure $\mu$ is (Ahlfors) Q-regular, that is, there is a constant $C>1$ such that

$$
C^{-1} r^{Q} \leq \mu(B(x, r)) \leq C r^{Q}
$$

for every $x \in X$ and $0<r<\operatorname{diam}(X)$, then the cocontent $\mathcal{H}_{R}^{d, \theta}$ is comparable (with two-sided inequalites) with the $(Q-d)$-dimensional NetrusovHausdorff content defined using the powers of radii.

A similar modification of the classical Hausdorff content is standard in the metric setting. The Hausdorff content of codimension $d, 0<d<\infty$, is

$$
\mathcal{H}_{R}^{d}(E)=\inf \left\{\sum_{j=1}^{\infty} \frac{\mu\left(B\left(x_{j}, r_{j}\right)\right)}{r_{j}^{d}}\right\},
$$

where $0<R<\infty$, and the infimum is taken over all coverings $\left\{B\left(x_{j}, r_{j}\right)\right\}$ of $E$ satisfying $r_{j} \leq R$ for all $j$. When $R=\infty$, the infimum is taken over all coverings $\left\{B\left(x_{j}, r_{j}\right)\right\}$ of $E$. Naturally, the Hausdoff measure of codimensiond is defined as

$$
\mathcal{H}^{d}(E)=\lim _{R \rightarrow 0} \mathcal{H}_{R}^{d}(E) .
$$

We use the following Leibniz type rule for fractional $s$-gradients, and its corollary, in the proofs of Theorems 5.4 and 5.5 . 
Lemma 5.2 ([HIT, Lemma 3.10 and Remark 3.11]). Let $0<s<1$, $0<p<\infty$ and $0<q \leq \infty$, and let $S \subset X$ be a measurable set. Let $u: X \rightarrow \mathbb{R}$ be a measurable function with $\left(g_{k}\right)_{k \in \mathbb{Z}} \in \mathbb{D}^{s}(u)$ and let $\varphi$ be a bounded L-Lipschitz function supported in $S$. Then sequences $\left(h_{k}\right)_{k \in \mathbb{Z}}$ and $\left(\rho_{k}\right)_{k \in \mathbb{Z}}$ where

$$
h_{k}=\left(g_{k}+2^{s k+2}|u|\right)\|\varphi\|_{\infty} \chi_{\operatorname{supp} \varphi}, \quad \rho_{k}=\left(g_{k}\|\varphi\|_{\infty}+2^{k(s-1)} L|u|\right) \chi_{\operatorname{supp} \varphi}
$$

are fractional s-gradients of $u \varphi$. Moreover, if $u \in N_{p, q}^{s}(S)$, then $u \varphi \in$ $N_{p, q}^{s}(X)$ and $\|u \varphi\|_{N_{p, q}^{s}(X)} \leq C\|u\|_{N_{p, q}^{s}(S)}$.

By choosing $u \equiv 1$ and $g_{k} \equiv 0$ for all $k \in \mathbb{Z}$ in (the proof of) the previous lemma, we obtain norm estimates for Lipschitz functions.

Corollary 5.3 ([ [IT, Corollary 3.12]). Let $0<s<1,0<p<\infty$ and $0<q \leq \infty$. Let $\varphi: X \rightarrow \mathbb{R}$ be an L-Lipschitz function supported in a bounded set $F \subset X$. Then $\varphi \in N_{p, q}^{s}(X)$ and

$$
\|\varphi\|_{N_{p, q}^{s}(X)} \leq C\left(1+\|\varphi\|_{\infty}\right)\left(1+L^{s}\right) \mu(F)^{1 / p},
$$

where the constant $C>0$ depends only on $s$ and $q$.

In the next theorem, we show that the Besov capacity of a set $E \subset X$ is bounded from above by a constant times the Netrusov-Hausdorff cocontent of $E$.

Theorem 5.4. Let $0<s<1,0<p<\infty, 0<q \leq \infty, E \subset X$ and $R \leq 1$. Then there is a constant $C>0$ such that

$$
C_{p, q}^{s}(E) \leq C \mathcal{H}_{R}^{s p, \theta}(E), \quad \text { where } \theta=\min \{1, q / p\} .
$$

Proof. Let $\left\{B\left(x_{j}, r_{j}\right)\right\}$ be a covering of the set $E$ such that $r_{j} \leq 1$ for all $j$. Let $i \in \mathbb{Z}_{+} \cup\{0\}$ and

$$
u_{i}(x)=\max \left\{0,1-2^{i} d\left(x, \bigcup_{j \in I_{i}} B\left(x_{j}, r_{j}\right)\right)\right\},
$$

where $I_{i}=\left\{j: 2^{-i} \leq r_{j}<2^{-i+1}\right\}$. Then $u_{i}=1$ in $\bigcup_{j \in I_{i}} B\left(x_{j}, r_{j}\right), u_{i}=0$ outside $\bigcup_{j \in I_{i}} B\left(x_{j}, 2^{-i+2}\right)$ and $u_{i}$ is Lipschitz with constant $2^{i}$. Since $i \geq 0$, we have $1+2^{i s} \leq C 2^{i s}$ and it follows from Corollary 5.3 and the doubling property that

$$
\begin{aligned}
C_{p, q}^{s}\left(\bigcup_{j \in I_{i}} B\left(x_{j}, r_{j}\right)\right) & \leq\left\|u_{i}\right\|_{N_{p, q}^{s}(X)}^{p} \\
& \leq C\left(1+\left\|u_{i}\right\|_{\infty}\right)^{p}\left(1+2^{i s}\right)^{p} \mu\left(\bigcup_{j \in I_{i}} B\left(x_{j}, 2^{-i+2}\right)\right) \\
& \leq C 2^{i s p} \mu\left(\bigcup_{j \in I_{i}} B\left(x_{j}, 2^{-i+2}\right)\right) \leq C \sum_{j \in I_{i}} \frac{\mu\left(B\left(x_{j}, r_{j}\right)\right)}{r_{j}^{s p}} .
\end{aligned}
$$


Let $\theta=\min \{1, q / p\}$. By Theorem 3.4 ,

$$
\begin{aligned}
C_{p, q}^{s}(E) & \leq C\left(\sum_{i} C_{p, q}^{s}\left(\bigcup_{j \in I_{i}} B\left(x_{j}, r_{j}\right)\right)^{\theta}\right)^{1 / \theta} \\
& \leq C\left(\sum_{i}\left(\sum_{j \in I_{i}} \frac{\mu\left(B\left(x_{j}, r_{j}\right)\right)}{r_{j}^{s p}}\right)^{\theta}\right)^{1 / \theta} ;
\end{aligned}
$$

the claim follows by taking the infimum over all covers $\left\{B\left(x_{j}, r_{j}\right)\right\}$ of $E$.

Next, we prove a converse result which gives a lower bound for the capacity in terms of the Netrusov-Hausdorff cocontent.

Theorem 5.5. Let $0<s<1,0<p<\infty, 0<q \leq \infty$ and let $\phi$ : $(0, \infty) \rightarrow(0, \infty)$ be an increasing function such that

$$
\int_{0}^{a} \phi(t)^{-1 / p} t^{s-1} d t<\infty \quad \text { for every } 0<a<\infty .
$$

Let $x_{0} \in X, 0<R<\infty$ and assume that $B\left(x_{0}, 8 R\right) \backslash B\left(x_{0}, 4 R\right)$ is nonempty. Then there are constants $C>0$ and $c>0$ such that

$$
\mathcal{H}_{c R}^{\phi, q / p}(E) \leq C C_{p, q}^{s}(E)
$$

for every compact set $E \subset B\left(x_{0}, R\right)$.

REMARK 5.6. For example, when $\phi(t)=t^{d}$, we have

$$
\int_{0}^{a} t^{-d / p+s-1} d t<\infty \text { if and only if } 1-s+d / p<1 .
$$

That is, $d<s p$.

Proof of Theorem 5.5. To avoid some inessential technical difficulties and make the notation simpler, we assume that $R=2^{-m}$ for $m \in \mathbb{Z}$. Our proof will show that the result for all $0<R<\infty$ can be obtained using the same argument.

Let $\varepsilon>0$ and $E \subset B\left(x_{0}, 2^{-m}\right)$ be a compact set. By Theorem 4.8, there is a locally Lipschitz function $v \in N_{p, q}^{s}(X)$ such that $v \geq 1$ on a neighbourhood of $E$ and

$$
\|v\|_{N_{p, q}^{s}(X)}^{p}<C C_{p, q}^{s}(E)+\varepsilon .
$$

Let $\psi$ be a Lipschitz function such that $\psi=1$ on $B\left(x_{0}, 2^{-m}\right)$ and $\psi=0$ outside $B\left(x_{0}, 2^{-m+1}\right)$. Then $u=v \psi \in N_{p, q}^{s}(X)$ is Lipschitz continuous and $u \geq 1$ on a neighbourhood of $E$. By Lemma 5.2 , there exists $\left(g_{k}\right)_{k \in \mathbb{Z}} \in \mathbb{D}^{s}(u)$ such that $g_{k}=0$ outside $B\left(x_{0}, 2^{-m+1}\right)$ for every $k$, and

$$
\left\|\left(g_{k}\right)\right\|_{l^{q}\left(L^{p}(X)\right)}^{p} \leq C\|v\|_{N_{p, q}^{s}(X)}^{p}<C\left(C_{p, q}^{s}(E)+\varepsilon\right) .
$$


To be precise, we have here the fractional $s$-gradient of $u$, still denoted by $g_{k}$, which satisfies the Sobolev-Poincare type inequality (4.1) that is later used in the proof. By 4.2$), g_{k}$ is supported in $B\left(x_{0}, 2^{-m+1}\right)$ for every $k$, and by (4.3) the inequality (5.2) is satisfied.

Let $x \in E$ be a generalized Lebesgue point of $u$ (see Definition 4.3). Since $u$ is continuous, it follows from Lemma 4.2 (h) that every point in $E$ is such a point. Then

$$
1 \leq u(x) \leq\left|u(x)-m_{u}^{\gamma}\left(B\left(x, 2^{-m}\right)\right)\right|+\left|m_{u}^{\gamma}\left(B\left(x, 2^{-m}\right)\right)\right| .
$$

We can estimate the first term by properties (d), (f) and (c) of the $\gamma$-median (Lemma 4.2), and by a telescoping argument

$$
\begin{aligned}
\left|u(x)-m_{u}^{\gamma}\left(B\left(x, 2^{-m}\right)\right)\right| & \leq \sum_{k \geq m}\left|m_{u}^{\gamma}\left(B\left(x, 2^{-k-1}\right)\right)-m_{u}^{\gamma}\left(B\left(x, 2^{-k}\right)\right)\right| \\
& \leq \sum_{k \geq m} m_{\left|u-m_{u}^{\gamma}\left(B\left(x, 2^{-k}\right)\right)\right|}^{\gamma}\left(B\left(x, 2^{-k-1}\right)\right) \\
& \leq \sum_{k \geq m} m_{\left|u-m_{u}^{\gamma}\left(B\left(x, 2^{-k}\right)\right)\right|}^{\gamma / C}\left(B\left(x, 2^{-k}\right)\right) .
\end{aligned}
$$

Hence, it follows from Theorem 4.5 and Remark 4.6 that

$$
\sum_{k \geq m} m_{\left|u-m_{u}^{\gamma}\left(B\left(x, 2^{-k}\right)\right)\right|}^{\gamma / C}\left(B\left(x, 2^{-k}\right)\right) \leq C \sum_{k \geq m} 2^{-k s}\left(\underset{B\left(x, 2^{-k+1}\right)}{f} g_{k}^{p} d \mu\right)^{1 / p} .
$$

Next, we estimate the second term of (5.3). Let $y \in B\left(x, 2^{-m}\right) \backslash F$, where $F$ is the exceptional set from Definition 2.1. Since $B\left(x, 2^{-m}\right) \subset B\left(x_{0}, 2^{-m+1}\right)$ and since $B\left(x_{0}, 2^{-m+3}\right) \backslash B\left(x_{0}, 2^{-m+2}\right)$ is nonempty, there exists $z \in$ $\left(B\left(x_{0}, 2^{-m+3}\right) \backslash B\left(x_{0}, 2^{-m+2}\right)\right) \backslash F$ such that $2^{-m} \leq d(y, z)<2^{-m+4}$. We define $g=\max \left\{g_{k}: m-4 \leq k \leq m-1\right\}$. Now,

$$
\begin{aligned}
|u(y)| & =|u(y)-u(z)| \\
& \leq d(y, z)^{s}(g(y)+g(z))=d(y, z)^{s} g(y) \leq 2^{(-m+4) s} g(y)
\end{aligned}
$$

and by (f), (b), (e) and (g) of Lemma 4.2 we have

$$
\begin{aligned}
\left|m_{u}^{\gamma}\left(B\left(x, 2^{-m}\right)\right)\right| & \leq m_{2^{(-m+4) s} g}^{\gamma}\left(B\left(x, 2^{-m}\right)\right) \leq C 2^{-m s} m_{g}^{\gamma}\left(B\left(x, 2^{-m}\right)\right) \\
& \leq C \sum_{k=m-4}^{m-1} 2^{-k s}\left(\underset{B\left(x, 2^{-m}\right)}{f} g_{k}^{p} d \mu\right)^{1 / p} \\
& \leq C \sum_{k \geq m} 2^{-k s}\left(\underset{B\left(x, 2^{-k+1}\right)}{f} g_{k}^{p} d \mu\right)^{1 / p} .
\end{aligned}
$$


Hence,

$$
\begin{aligned}
& 1 \leq C \sum_{k \geq m} 2^{-k s}\left(\underset{B\left(x, 2^{-k+1}\right)}{f} g_{k}^{p} d \mu\right)^{1 / p} \\
& \leq C\left(\sum_{k \geq m} \phi\left(2^{-k+1}\right)^{-1 / p} 2^{-k s}\right) \sup _{k \geq m} \phi\left(2^{-k+1}\right)^{1 / p}\left(f_{B\left(x, 2^{-k+1}\right)} g_{k}^{p} d \mu\right)^{1 / p} \\
& \leq C\left(\int_{0}^{2^{-m+1}} \phi(t)^{-1 / p} t^{s-1} d t\right) \sup _{k \geq m} \phi\left(2^{-k+1}\right)^{1 / p}\left(\underset{B\left(x, 2^{-k+1}\right)}{f} g_{k}^{p} d \mu\right)^{1 / p} \\
& \leq C \sup _{k \geq m} \phi\left(2^{-k+1}\right)^{1 / p}\left(\underset{B\left(x, 2^{-k+1}\right)}{f} g_{k}^{p} d \mu\right)^{1 / p} .
\end{aligned}
$$

Now, for every $x \in E$, there is a ball $B\left(x, 2^{-k_{x}+1}\right)$, such that

$$
\frac{\mu\left(B\left(x, 2^{-k_{x}+1}\right)\right)}{\phi\left(2^{-k_{x}+1}\right)} \leq C \int_{B\left(x, 2^{-k_{x}+1}\right)} g_{k_{x}}^{p} d \mu .
$$

By the $5 r$-covering lemma, there exists a countable family of disjoint balls $B_{j}=B\left(x_{j}, 2^{-k_{x_{j}}+1}\right)$, of radii $r_{j}=2^{-k_{x_{j}}+1} \leq 2^{-m}$, such that the dilated balls $5 B_{j}$ cover the set $E$. We write $j \in I_{i}$ when $2^{-i} \leq 5 r_{j}<2^{-i+1}$. Then $k_{x_{j}}=i+3$ for $j \in I_{i}$, and since $\phi$ is increasing we see that

$$
\sum_{j \in I_{i}} \frac{\mu\left(5 B_{j}\right)}{\phi\left(5 r_{j}\right)} \leq C \sum_{j \in I_{i}} \frac{\mu\left(B_{j}\right)}{\phi\left(r_{j}\right)} \leq C \sum_{j \in I_{i}} \int_{B_{j}} g_{i+3}^{p} d \mu \leq C\left\|g_{i+3}\right\|_{L^{p}(X)}^{p},
$$

where we have also used doubling and the disjointness of the balls $B_{j}$. Summing over $i$, we obtain

$$
\sum_{2^{-i}<5 \cdot 2^{-m}}\left(\sum_{j \in I_{i}} \frac{\mu\left(5 B_{j}\right)}{\phi\left(5 r_{j}\right)}\right)^{q / p} \leq C \sum_{i \in \mathbb{Z}}\left\|g_{i+3}\right\|_{L^{p}(X)}^{q},
$$

and it follows that

$$
\mathcal{H}_{5 \cdot 2^{-m}}^{\phi, q / p}(E) \leq C\left(\sum_{i \in \mathbb{Z}}\left\|g_{i+3}\right\|_{L^{p}(X)}^{q}\right)^{p / q} .
$$

Now, letting $\epsilon \rightarrow 0$ in 5.2 proves the claim.

Acknowledgments. I am grateful to Toni Heikkinen for helpful comments and ideas during the preparation of this work. This research was supported by the Academy of Finland (grant no. 272886).

\section{References}

[A1] D. R. Adams, The classification problem for the capacities associated with the Besov and Triebel-Lizorkin spaces, in: Approximation and Function Spaces (Warszawa, 1986), Banach Center Publ. 22, PWN, Warszawa, 1989, 9-24. 
[A2] D. R. Adams, Besov capacity redux, J. Math. Sci. (N.Y.) 162 (2009), 307-318.

[AH] D. R. Adams and L. I. Hedberg, Function Spaces and Potential Theory, Grundlehren Math. Wiss. 314, Springer, Berlin, 1996.

[AHS] D. R. Adams and R. Hurri-Syrjänen, Besov functions and vanishing exponential integrability, Illinois J. Math. 47 (2003), 1137-1150.

[AX] D. R. Adams and J. Xiao, Strong type estimates for homogeneous Besov capacities, Math. Ann. 325 (2003), 695-709.

[BB] A. Björn and J. Björn, Nonlinear potential theory on metric spaces, EMS Tracts Math. 17, Eur. Math. Soc., Zürich, 2011.

[B] M. Bourdon, Une caractérisation algébrique des homéomorphismes quasi-Möbius, Ann. Acad. Sci. Fenn. Math. 32 (2007), 235-250.

[CW] R. R Coifman et G. Weiss, Analyse harmonique non-commutative sur certains espaces homogènes, Lecture Notes in Math. 242, Springer, Berlin, 1971.

[Co] Ş. Costea, Besov capacity and Hausdorff measures in metric measure spaces, Publ. Mat. 53 (2009), 141-178.

[D] J. R. Dorronsoro, On the differentiability of Lipschitz-Besov functions, Trans. Amer. Math. Soc. 303 (1987), 229-240.

[F] N. Fujii, A condition for a two-weight norm inequality for singular integral operators, Studia Math. 98 (1991), 175-190.

[GKS] A. Gogatishvili, P. Koskela and N. Shanmugalingam, Interpolation properties of Besov spaces defined on metric spaces, Math. Nachr. 283 (2010), 215-231.

[GKZ] A. Gogatishvili, P. Koskela and Y. Zhou, Characterizations of Besov and TriebelLizorkin spaces on metric measure spaces, Forum Math. 25 (2013), 787-819.

[GT] V. Gol'dshtein and M. Troyanov, Capacities in metric spaces, Integral Equations Operator Theory 44 (2002), 212-242.

[H] P. Hajłasz, Sobolev spaces on an arbitrary metric space, Potential Anal. 5 (1996), 403-415.

[HK] H. Hakkarainen and J. Kinnunen, The BV-capacity in metric spaces, Manuscripta Math. 132 (2010), 369-390.

[HMY] Y. Han, D. Müller, and D. Yang, A theory of Besov and Triebel-Lizorkin spaces on metric measure spaces modeled on Carnot-Carathéodory spaces, Abstr. Appl. Anal. (2008), art. ID 893409, 250 pp.

[HN] L. I. Hedberg and Yu. Netrusov, An axiomatic approach to function spaces, spectral synthesis, and Luzin approximation, Mem. Amer. Math. Soc. 188 (2007), no. 882.

[HIT] T. Heikkinen, L. Ihnatsyeva and H. Tuominen, Measure density and extension of Besov and Triebel-Lizorkin functions, J. Fourier Anal. Appl. 22 (2016), 334-382.

[HKT] T. Heikkinen, P. Koskela and H. Tuominen, Approximation and quasicontinuity of Besov and Triebel-Lizorkin functions, arXiv:1505.05680 (2015).

[HT1] T. Heikkinen and H. Tuominen, Smoothing properties of the discrete fractional maximal operator on Besov and Triebel-Lizorkin spaces, Publ. Mat. 58 (2014), 379-399.

[HT2] T. Heikkinen and H. Tuominen, Approximation by Hölder functions in Besov and Triebel-Lizorkin spaces, arXiv:1504.02585 (2015).

[JPW] B. Jawerth, C. Pérez and G. Welland, The positive cone in Triebel-Lizorkin spaces and the relation among potential and maximal operators, in: Harmonic Analysis and Partial Differential Equations (Boca Raton, FL, 1988), Contemp. Math. 107, Amer. Math. Soc., Providence, RI, 1990, 71-91.

[JT] B. Jawerth and A. Torchinsky, Local sharp maximal functions, J. Approx. Theory 43 (1985), 231-270.

[K] T. Kilpeläinen, A remark on the uniqueness of quasicontinuous functions, Ann. Acad. Sci. Fenn. Math. 23 (1998), 261-262. 
[KL] J. Kinnunen and V. Latvala, Lebesgue points for Sobolev functions on metric spaces, Rev. Mat. Iberoamer. 18 (2002), 685-700.

[KM] J. Kinnunen and O. Martio, The Sobolev capacity on metric spaces, Ann. Acad. Sci. Fenn. Math. 21 (1996), 367-382.

[KYZ] P. Koskela, D. Yang and Y. Zhou, Pointwise characterizations of Besov and TriebelLizorkin spaces and quasiconformal mappings, Adv. Math. 226 (2011), 3579-3621.

[Leh] J. Lehrbäck, Neighbourhood capacities, Ann. Acad. Sci. Fenn. Math. 37 (2012), $35-51$.

[Ler] A. K. Lerner, A pointwise estimate for the local sharp maximal function with applications to singular integrals, Bull. London Math. Soc. 42 (2010), 843-856.

[LP] A. K. Lerner and C. Pérez, Self-improving properties of generalized Poincaré type inequalities through rearrangements, Math. Scand. 97 (2005), 217-234.

[MS] R. A. Macías and C. Segovia, A decomposition into atoms of distributions on spaces of homogeneous type, Adv. Math. 33 (1979), 271-309.

[MX] M. Milman and J. Xiao, The $\infty$-Besov capacity problem, arXiv:1506.01901 (2015).

[MY] D. Müller and D. Yang, A difference characterization of Besov and Triebel-Lizorkin spaces on RD-spaces, Forum Math. 21 (2009), 259-298.

[N1] Yu. V. Netrusov, Sets of singularities of functions in spaces of Besov and LizorkinTriebel type, Trudy Mat. Inst. Steklova 187 (1989), 162-177 (in Russian); English transl.: Proc. Steklov Inst. Math. 1990, 185-203.

[N2] Yu. V. Netrusov, Metric estimates for the capacities of sets in Besov spaces, Trudy Mat. Inst. Steklova 190 (1989), 159-185 (in Russian); English transl.: Proc. Steklov Inst. Math. 1992, 167-192.

[N3] Yu. V. Netrusov, Estimates of capacities associated with Besov spaces, Zap. Nauchn. Sem. S.-Peterburg. Otdel. Mat. Inst. Steklova (POMI) 201 (1992), 124-156 (in Russian); English transl.: J. Math. Sci. 78 (1996), 199-217.

[NS] J. Nuutinen and P. Silvestre, The Riesz capacity in metric spaces, arXiv:1501.05746 (2015).

[PT] J. Poelhuis and A. Torchinsky, Medians, continuity, and vanishing oscillation, Studia Math. 213 (2012), 227-242.

[SYY] N. Shanmugalingam, D. Yang and W. Yuan, Newton-Besov spaces and NewtonTriebel-Lizorkin spaces on metric measure spaces, Positivity 19 (2015), 177-220.

[Sto] B.-M. Stocke, Differentiability properties of Bessel potentials and Besov functions, Ark. Mat. 22 (1984), 269-286.

[Str] J.-O. Strömberg, Bounded mean oscillation with Orlicz norms and duality of Hardy spaces, Indiana Univ. Math. J. 28 (1979), 511-544.

[Y] D. Yang, New characterizations of Hajtasz-Sobolev spaces on metric spaces, Sci. China Ser. A 46 (2003), 675-689.

[YZ] D. Yang and Y. Zhou, New properties of Besov and Triebel-Lizorkin spaces on RD-spaces, Manuscripta Math. 134 (2011), 59-90.

[Z] Y. Zhou, Fractional Sobolev extension and imbedding, Trans. Amer. Math. Soc. 367 (2015), 959-979.

Juho Nuutinen

Department of Mathematics and Statistics

P.O. Box 35

University of Jyväskylä, FI-40014, Finland

E-mail: juho.nuutinen@jyu.fi 